\title{
Adaptive OFDM-Based Acoustic Underwater Transmission: System Design and Experimental Verification
}

\author{
Mohammad Sadeghi, Mohammed Elamassie and Murat Uysal \\ Department of Electrical and Electronics Engineering, Ozyegin University, Istanbul, Turkey, 34794 \\ E-mail: mohammad.sadeghi@ozu.edu.tr
}

\begin{abstract}
In this paper, we present the design and implementation of a software defined orthogonal frequency division multiplexing (OFDM)-based underwater acoustic (UWA) communication system with link adaptation. Our system implementation is based on the customized versions of National Instruments Universal Software Radio Peripheral (USRP). The modified USRPs are interfaced with hydrophone front-ends for acoustic transmission. We investigate the performance of various adaptive algorithms where both modulation order/type and power on each subcarrier are selected based on channel conditions in order to maximize throughput. The experimental in-pool test results verify the superiority of adaptive transmission.
\end{abstract}

Index Terms-Adaptive transmission, link adaptation, orthogonal frequency division multiplexing (OFDM), underwater acoustic communication.

\section{INTRODUCTION}

Emerging underwater applications such as offshore oil field exploration/monitoring, oceanographic data collection, maritime archaeology, environmental monitoring, disaster prevention, port security etc. require the deployment of wireless links instead of conventional wireline links [1], [2]. While radio and optical links can be possibly used underwater, acoustic transmission is widely deployed particularly due to long range transmission capability.

Underwater acoustic (UWA) modems have ranges up to several kilometers albeit at relatively low data rates. In an effort to improve link reliability and boost data rates, link adaptation can be used that involves the selection of transmission parameters according to channel conditions. While adaptive transmission techniques have been widely investigated for radio frequency (RF) channels, see [3], the current results for adaptive UWA systems are rather limited. The studies in [4]-[8] rely on simulated channel and recorded data from earlier sea trials. On the other hand, the works in [9]-[13] provide results for adaptive UWA systems based on experiments conducted either in pool or sea. In [9], an adaptive modulation and coding (AMC) technique is presented to maximize the data rate and the verification of the proposed technique takes place as a part of AUV Fest 2007 experiment. In [10], two adaptive UWA algorithms are proposed inspired from the greedy algorithms earlier introduced in [14], [15] for RF communications and their performances are tested during the KAM11 experiment. Aiming to maximize the spectral efficiency, the first algorithm of [10] adjusts the modulation level while the second one adjusts both the modulation level and the power on each sub-carrier. The experimental works in [11], [12] are conducted by employing AquaSeNT commercial OFDM modem [16]. In [11], the performance of AMC is studied for this modem's available modulation and coding pairs, i.e., BPSK, QPSK, 16-QAM and LDPC codes with rates of $1 / 2$ and $3 / 4$. The work is further extended to a multiuser scenario in [12].

The existing experimental works on adaptive UWA OFDM systems are either based on the commercial modems [11], [12] or conducted as a part of the large UWA measurement campaigns [9], [10]. Such traditional hardware devices limit cross-functionality and can only be modified through physical intervention. On the contrary, software defined platforms provide efficient, relatively easy to implement and inexpensive solutions, where some or all of the PHY functionalities are implemented through modifiable software or firmware operating on programmable processing technologies. This allows new wireless features and capabilities to be added to existing systems without requiring new hardware.

To the best of our knowledge, the only experimental work that investigates adaptive UWA transmission on a software defined platform is reported in [13]. A modified software defined radio (SDR) platform (Ettus USRP N210) is employed in [13] and the performance of adaptive modulation is investigated through lake experiments. In our work, we adopt a similar approach and use National Instruments (NI) USRP 2930 where the RF cards are replaced by LFTX/LFRX baseband daughter boards. The modified USRPs are interfaced with hydrophone front-ends to create a software defined UWA experimental setup. Unlike [13] where only adaptive modulation is investigated (i.e., fixed modulation size is selected for all subcarriers), we investigate the performance of more advanced adaptive algorithms where both modulation order/type and power on each subcarrier are selected based on channel conditions to maximize throughput. We experimentally verify the performance of these adaptive algorithms on our customized software defined UWA platform and demonstrate the superiority of adaptive transmission.

The rest of the paper is organized as follows. In Section II, we introduce the overall system architecture followed by the description of adaptive algorithms under consideration. In Section III, we present the description of experimental set-up. In Section IV, we provide the experimental results obtained in the pool. Finally, we provide concluding remarks in Section V. 


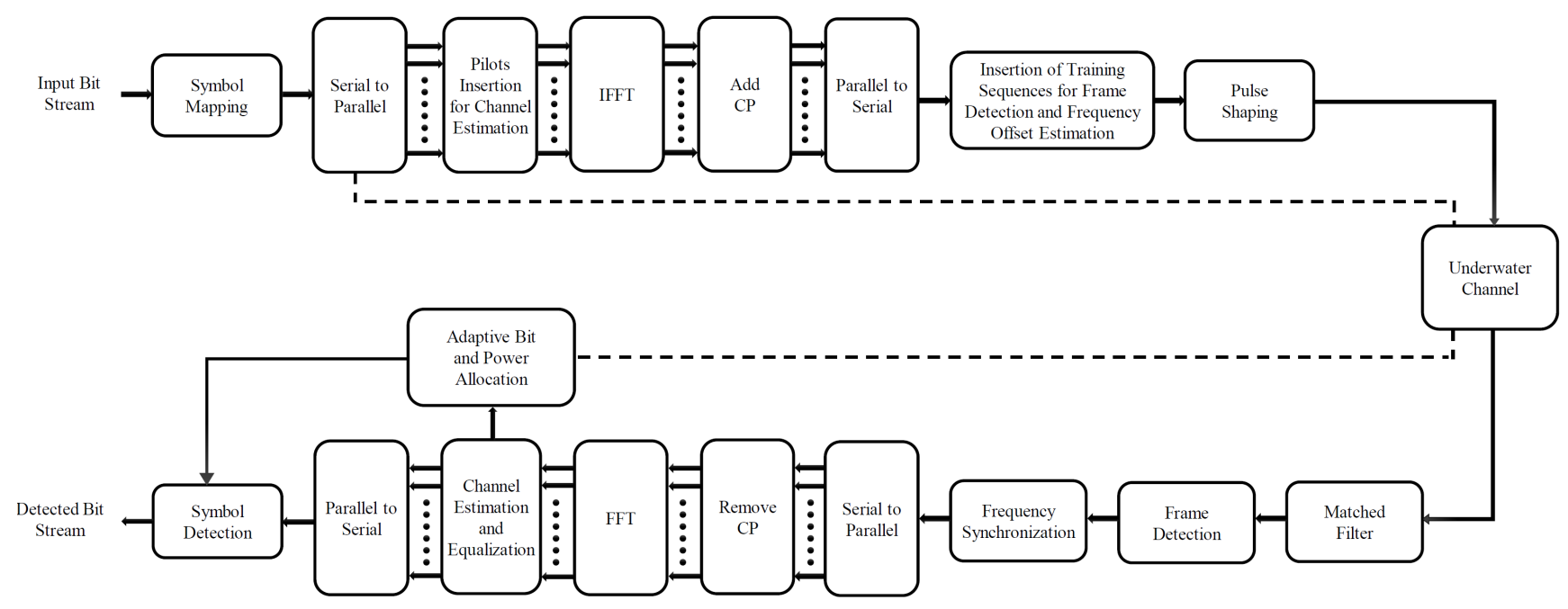

Fig. 1. System overview

\section{SySTEM MODEL}

\section{A. Overall Architecture}

The block diagram of the adaptive OFDM system under consideration is illustrated in Fig. 1. The transmitter first sends a known training sequence to estimate the channel. In our implementation, the training sequence is arranged in block type form [17]. In this arrangement, pilot symbols are transmitted periodically in which all subcarriers are used as pilot symbols. At the receiver side, the training sequence is extracted via Least Square (LS) estimation. Based on the channel state information (CSI), the transmission parameters, i.e., modulation type/size and power level on each subcarrier, are selected by the adaptive transmission algorithms (see Section II.B for further details). Then, this information is fed back to transmitter side (see Section II.C for additional details). Based on this received feedback, modulation sizes and power levels are assigned to subcarriers. Followed by the training phase, information data is then transmitted to the receiver.

We employ an OFDM system with $K$ subcarriers. The input bit stream is first mapped to the modulation symbols. The adopted modulation types/sizes are BPSK, 4-QAM, 8-QAM and 16-QAM. $K$-point Inverse Fast Fourier Transform (IFFT) is applied to the modulation symbols and a cyclic prefix (CP) is appended to eliminate the intersymbol-interference (ISI) caused by multipath effects. At the beginning of each OFDM frame, one training sequence for frame detection and another training sequence for frequency offset estimation are added. Frames are separated from each other by a guard interval. After pulse shaping, the resulting signal is transmitted in the underwater channel. At the receiver side, the received signal is first convolved with known training sequence and the position of peak determines the beginning of frame. After frame synchronization, the Schmidl-Cox algorithm [18] is applied for frequency synchronization. Followed by the CP elimination, the resulting sequence is applied to Fast Fourier Transform (FFT). The received signal on the $k^{\text {th }}$ subcarrier in frequency domain is written as

$$
Y_{k}=\sqrt{P_{k}} \widehat{H}_{k} X_{k}+N_{k}
$$

where $P_{k}, \widehat{H}_{k}$ and $N_{k}$ respectively denote the transmitted power,the estimated channel gain and complex additive noise/interference at $k^{\text {th }}$ subcarrier. This is followed by LS channel estimation and one tap equalizer. Finally, the equalized data symbols are demodulated and the information data is recovered.

\section{B. Adaptive Algorithms}

We consider three different adaptive algorithms which were originally proposed in the context of RF communications [14], [15] and also considered for UWA communications in [10]. In Adaptive Algorithm 1, average throughput is maximized by assigning the highest modulation on each subcarrier in a manner not to exceed the targeted bit error rate (BER) on that subcarrier. In Adaptive Algorithm 2, bit loading is done in a way to maximize throughput while satisfying a targeted average BER. In this algorithm, bit loading is initiated by assigning the first bit to the subcarrier that imposes the least BER to accept one bit. The modulation levels are then increased for those subcarriers that require the least BER increment and the algorithm stops when the target BER is achieved. While the previous two adaptive algorithms rely on adaptive modulation, adaptive selection of modulation and power level is considered in the third one. Specifically, in Adaptive Algorithm 3, throughput is maximized by adaptive allocation of the modulation size and power level of each subcarrier while maintaining the BER at a specific threshold. Similar to the previous algorithm, a greedy algorithm is used to allocate modulation sizes and power levels. Bits are assigned to the subcarriers that need the least increase in power to reach the next modulation size. No data is transmitted on the subcarriers that experience deep fade (i.e., zero power is allocated). The algorithm stops when the total power is exceeded, or when all subcarriers achieve the highest modulation level (i.e., 16-QAM in our case). The flowchart of the algorithms are available in [14], [15]. 


\section{Feedback Information}

In the feedback phase, to ensure a reliable transmission, channel coding is employed. Specifically, we employ a 4 state convolutional encoder with a rate of $1 / 2$ [19]. In Adaptive Algorithms 1 and 2, we use three bits to represent the selected modulation level. The constructed bit stream is then encoded by convolutional coding and mapped to QPSK symbols. In Adaptive Algorithm 3, in addition to modulation size, power levels are selected and mapped to 16 quantization levels. The quantization level is represented with four bits, then encoded by convolutional coding and mapped to QPSK symbols.

\section{EXPERIMENTAL SET-UP}

Fig. 2 shows the block diagram of our experimental setup. At the transmitter side, the laptop computer generates adaptive OFDM symbols using LabVIEW. The data is transferred with Ethernet cable from the laptop to our customized software defined platform (NI USRP 2930). The RF cards in the USRPs are replaced by LFTX/LFRX baseband daughter boards with output sampling rate up to $5 \mathrm{MHz}$.

The signal transmitted by the NI USRP is further amplified by a power amplifier (GST YE5873A) to adjust the signal power for the input of acoustic hydrophone. The hydrophones are commercially available from Teledyne (Reson TC4040/TC4033) that are omni-directional and suitable for an operational frequency range from $1 \mathrm{~Hz}$ to $170 \mathrm{KHz}$. At the receiver, signal is received by hydrophone followed by a pre-amplifier (Reson VP1000). The received analog signal at the USRP is sampled and digitized to produce a digital signal which is then transferred to the laptop. The received signal is processed in LabVIEW to detect the transmitted data.

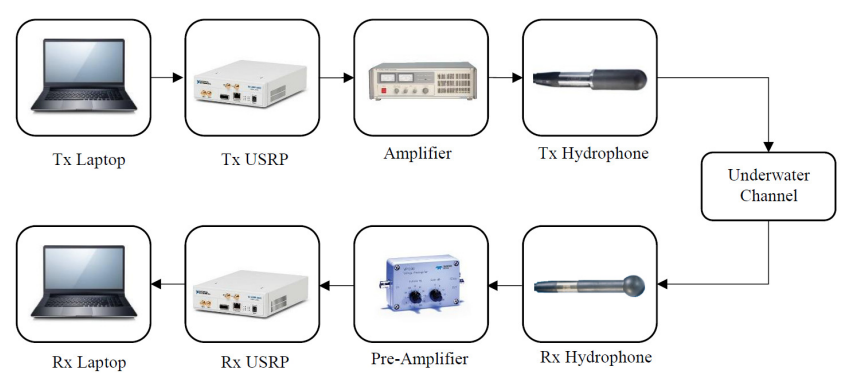

Fig. 2. Block diagram of experimental set-up

\section{PoOl ExPERIMENT Results}

In this section, we present the real-time experimental results obtained from pool trials to evaluate the performance of the designed adaptive OFDM system. The transmitter and receiver are submerged in the pool at a depth of 2 meters. The distance between transmitter and receiver is varied between 0.5 to 2 meters (See Fig.3). The system parameters are summarized in Table I.

As an example to demonstrate real-time transmission, we transmit a specific OFDM symbol (that carries a generated PN sequence) with 64 subcarriers at a transmit power of 24 $\mathrm{dB}$. The distance is 0.5 meter. The targeted average BER is selected as $0.05^{1}$. Fig.4.a. illustrates the loaded bits on

${ }^{1}$ In [13], a BER of 0.05 is used for tank experiment while a BER of 0.001 is used for lake experiment. In [10], the targeted BER range varies within 0.001-0.01 for sea experiments.
TABLE I. System parameters

\begin{tabular}{|c|c|}
\hline Parameters & Values \\
\hline Number of Subcarriers & $K=64,128$ \\
\hline Carrier Frequency & $30 \mathrm{KHz}$ \\
\hline Modulation & BPSK/ 4-QAM/ 8-QAM/ 16-QAM \\
\hline Pulse Shaping & Raised cosine $(\beta=0.5)$ \\
\hline Bandwidth & $4 \mathrm{KHZ}$ \\
\hline Guard Time & $15 \mathrm{~ms}$ \\
\hline Feedback Length & 3,7 OFDM symbols \\
\hline
\end{tabular}

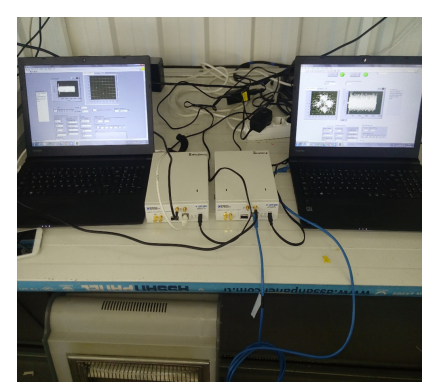

(a)

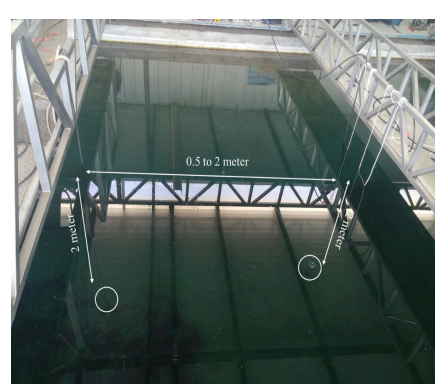

(b)
Fig. 3. (a) SDR setup (b) pool environment

each subcarrier for each algorithm under consideration. It can be observed that Adaptive Algorithm 3 achieves higher modulation sizes compared to other algorithms as a result of
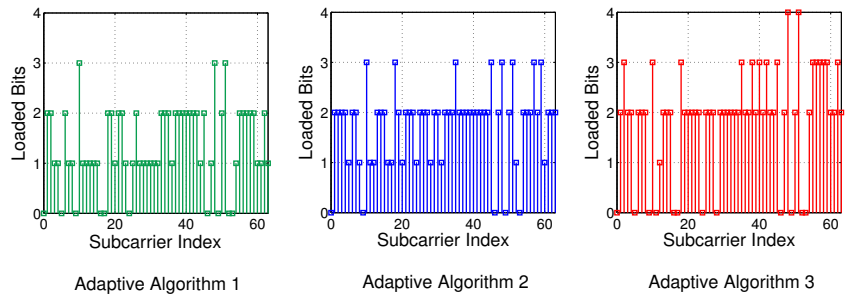

(a)

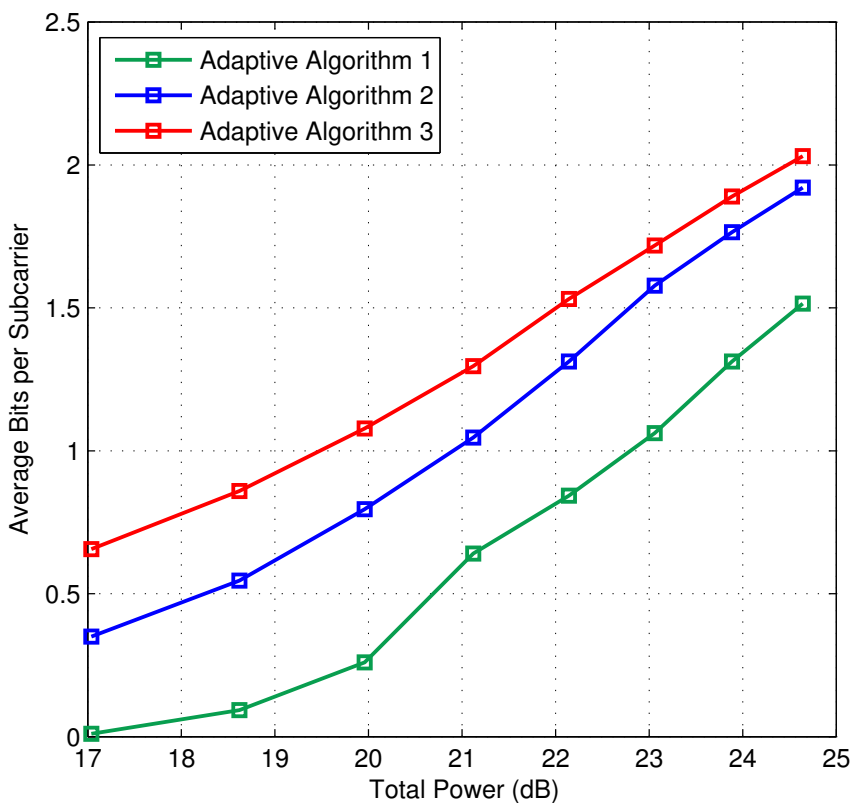

(b)

Fig. 4. (a) Loaded bits on each subcarrier for Adaptive Algorithm 1, 2 and 3. (b) Average bits per subcarrier versus total transmitted power in $\mathrm{dB}$ 


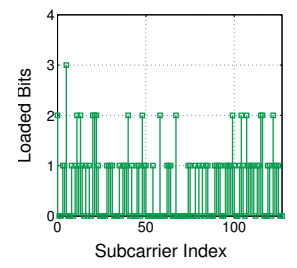

Adaptive Algorithm 1

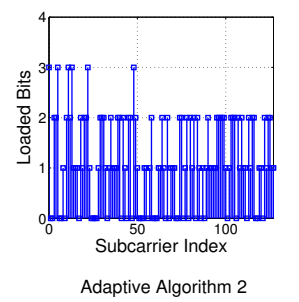

(a)

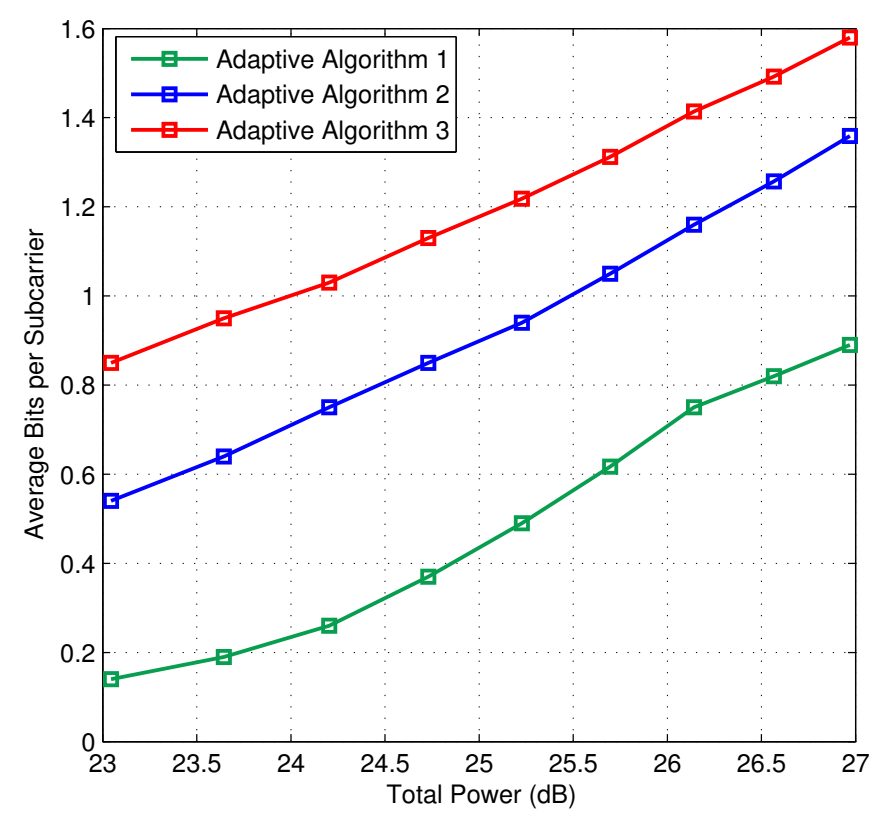

(b)
Fig. 5. (a) Loaded bits on each subcarrier for Adaptive Algorithm 1, 2 and 3. (b) Average bits per subcarrier versus total transmitted power in $\mathrm{dB}$

power adaptation. In Fig.4.b, we present average achievable throughput (in bits per subcarrier) by transmitting 10 packets of OFDM symbols with 500 bits per each packet. It is observed that Adaptive Algorithm 3 outperforms Adaptive Algorithm 2 for the considered range of total power. This performance difference decreases as the total power increases, since Algorithm 3 tends to distribute power among subcarriers more equally in high total powers regime resulting in a similar performance with Algorithm 2.

In Fig. 5, we repeat the throughput test at the distance of 1 meter with 128 subcarriers. It can be noticed that Adaptive Algorithm 3 achieves higher modulation due to its ability to adapt power on each subcarrier. Comparing to the previous results, it can be observed that the system achieves lower throughputs as the distance increases due to higher attenuation and more severe multipath effects.

In Fig. 6, we compare the BER of adaptive OFDM system (assuming the use of Adaptive Algorithm 2) with a nonadaptive OFDM system (i.e., with fixed modulation type regardless of channel conditions). 4-QAM is deployed for the non-adaptive system. 12 consecutive packets of PN sequence bits are transmitted. It is observed from Fig. 6 that unlike nonadaptive system, in an adaptive system, subcarriers can accept different modulation sizes with relatively lower average BER. In Fig. 7, we present the averaged bits per subcarrier versus

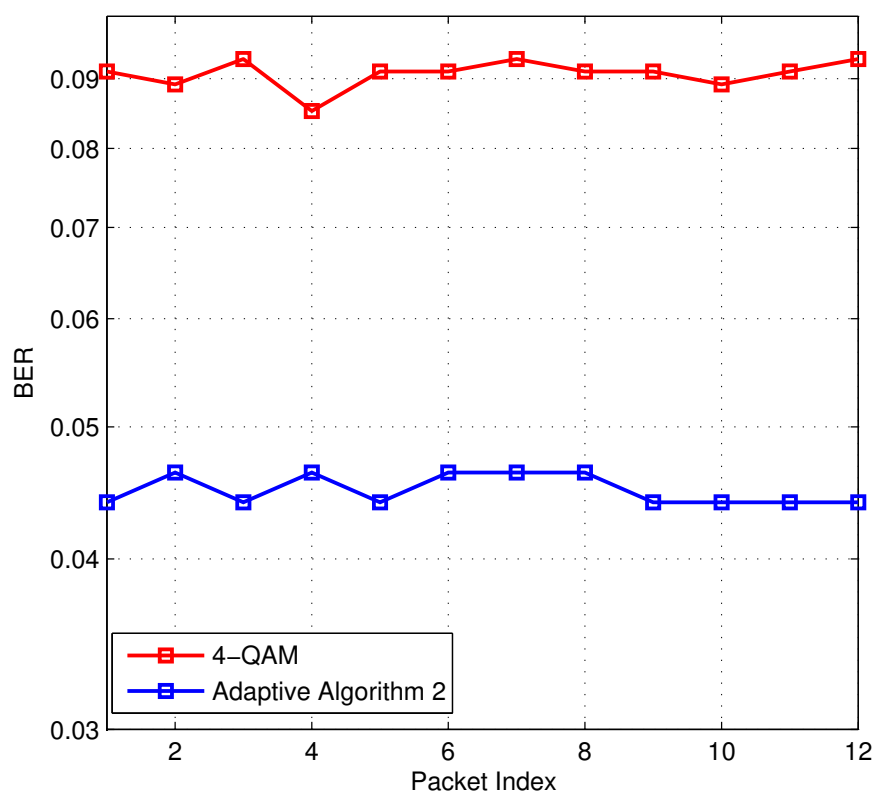

Fig. 6. BER comparison of fixed and Adaptive Algorithm 2

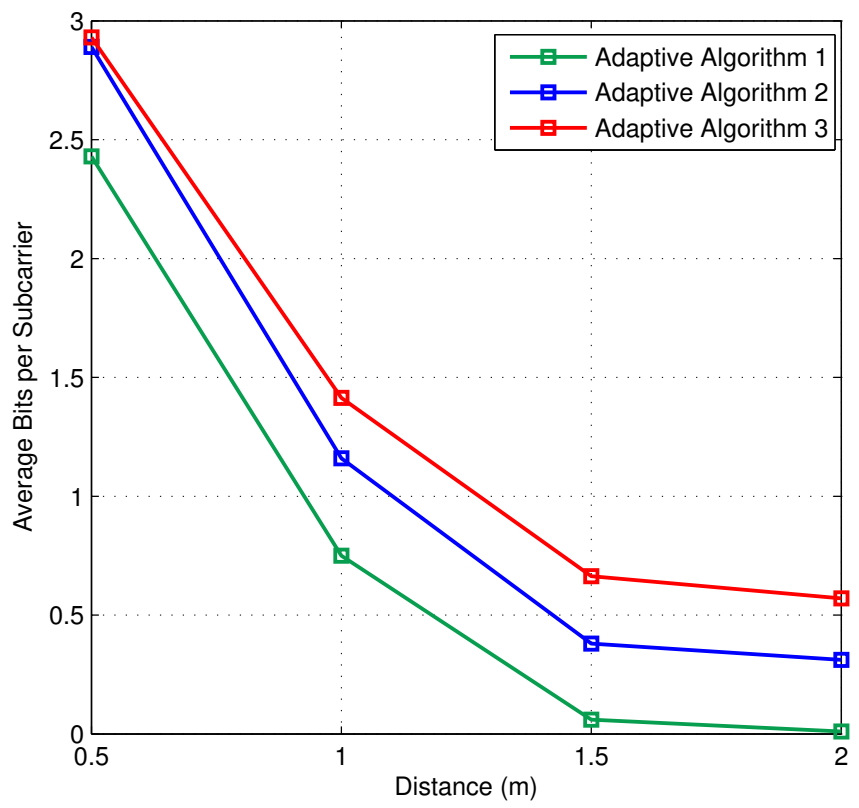

Fig. 7. Average bits per subcarrier versus distance at the transmit power of $26 \mathrm{~dB}$

distance. The transmit power is $26 \mathrm{~dB}$. It can be observed that the achieved throughput decreases as the distance increases due to attenuation and more severe multipath effects.

\section{CONCLUSIONS}

In this paper, we presented the design and implementation of an adaptive OFDM-based UWA communication system on a customized software defined platform. The physical layer was built upon adaptive algorithms where modulation size-type and/or power level were selected based on channel conditions. The adaptive OFDM system was tested in pool and its superior performance over non-adaptive counterparts was demonstrated. 


\section{REFERENCES}

[1] S. Zhou and Z. Wang, OFDM for underwater acoustic communications. John Wiley and Sons, Inc., 2014.

[2] S. Al-Dharrab, M. Uysal, and T. M. Duman, "Cooperative underwater acoustic communications," IEEE Communications Magazine, vol. 51 no. 7 , pp. 146-153, 2013.

[3] A. J. Goldsmith and S.-G. Chua, "Variable-rate variable-power MQAM for fading channels," IEEE Transactions on Communications, vol. 45, no. 10, pp. 1218-1230, 1997.

[4] P. Qarabaqi and M. Stojanovic, "Adaptive power control for underwater acoustic communications," in IEEE OCEANS, pp. 1-7, 2011

[5] A. Pottier, F. X. Socheleau, and C. Laot, "Distributed power allocation strategy in shallow water acoustic interference channels," in 17th International Workshop on Signal Processing Advances in Wireless Communications (SPAWC), pp. 1-5, 2016.

[6] B. Tomasi, L. Toni, L. R. P. Casari, and M. Zorzi, "Performance study of variable-rate modulation for underwater communications based on experimental data," in MTS/IEEE OCEANS, pp. 1-8, 2010.

[7] X. Huang and V. B. Lawrence, "Bandwidth-efficient bit and power loading for underwater acoustic OFDM communication system with limited feedback," in IEEE 73rd Vehicular Technology Conference (VTC Spring), pp. 1-5, 2011.

[8] K. Pelekanakis, L. Cazzanti, G. Zappa, and J. Alves, "Decision treebased adaptive modulation for underwater acoustic communications," in IEEE Third Underwater Communications and Networking Conference (UComms), pp. 1-5, 2016.

[9] M. Sanjay, T. M. Duman, and P. Hursky, "Adaptive coding/modulation for shallow-water uwa communications," in Acoustic Conference, pp. 15, 2008.

[10] A. Radosevic, R. Ahmed, T. M. Duman, J. G. Proakis, and M. Stojanovic, "Adaptive OFDM modulation for underwater acoustic com- munications: Design considerations and experimental results," IEEE Journal of Oceanic Engineering, vol. 39, no. 2, pp. 357-370, 2014.

[11] L. Wan, H. Zhou, X. Xu, Y. Huang, S. Zhou, Z. Shi, and J. H. Cui, "Adaptive modulation and coding for underwater acoustic OFDM," IEEE Journal of Oceanic Engineering, vol. 40, no. 2, pp. 327-336, 2015.

[12] Y. Zhang, Y. Huang, L. Wan, X. S. S. Zhou, and H. Wang, "Adaptive OFDMA with partial csi for downlink underwater acoustic communications," Journal of Communications and Networks, vol. 18, no. 3, pp. 387-396, 2016.

[13] E. Demirors, G. Sklivanitis, G. E. Santagati, T. Melodia, and S. N. Batalama, "Design of a software-defined underwater acoustic modem with real-time physical layer adaptation capabilities," in ACM International Conference on Underwater Networks Systems (WUWNet), pp. 1$8,2014$.

[14] P. Xia, S. Zhou, and G. B. Giannakis, "Adaptive MIMO-OFDM based on partial channel state information," IEEE Transactions on Signal Processing, vol. 52, no. 1, pp. 202-213, 2004.

[15] R. F. H. Fischer and J. B. Huber, "A new loading algorithm for discrete multitone transmission," in Global Telecommunications Conference, GLOBECOM '96., pp. 724-728, 1996.

[16] AquaSeNT, "Underwater acoustic OFDM modem." http://www. aquasent.com.

[17] M. Coleri, M. Ergen, A. Puri, and A. Bahai, "Channel estimation techniques based on pilot arrangement in OFDM systems," IEEE Transactions on Broadcasting, vol. 48, no. 3, pp. 223-229, 2002.

[18] T. M. Schmidl and D. C. Cox, "Robust frequency and timing synchronization for OFDM," IEEE Transactions on Communications, vol. 45 , no. 12, pp. 1613-1621, 1997.

[19] R. Blahut, Algebraic codes for data transmission. Cambridge, U.K.: Cambridge University Press, 2003. 